\title{
Bee product efficacy in children with upper respiratory tract infections
}

\author{
Yılmaz Seçilmişş ${ }^{1 \oplus}$ Sibel Silici ${ }^{2 \oplus}$ \\ ${ }^{1}$ Division of Pediatric Emergency, Department of Pediatrics, Erciyes University Faculty of Medicine, Kayseri; ${ }^{2}$ Department of \\ Agricultural Biotechnology, Erciyes University Faculty of Agriculture, Kayseri, Turkey.
}

\begin{abstract}
Background and objectives. The most common infectious disease in children is acute upper respiratory tract infection (URTI). Many drugs, especially antitussive drugs, are used for symptomatic treatment. Bee products (propolis, royal jelly, and honey) have antiviral, antibacterial, and antioxidant properties, and they have synergistic effects with antibiotics. The aim of this study was to evaluate the effectiveness of a mixture of bee products in URTI in children.
\end{abstract}

Methods. The patients were divided into four groups consisting of two bacterial groups receiving either antibiotics or antibiotics + bee products and two viral groups treated with either placebo or bee products. Disease severity and improvement duration were assessed by the Canadian Acute Respiratory Illness and Flu Scale (CARIFS) Score.

Results. One hundred and four patients (59 male, 56.7\%; 45 female, 43.3\%) aged between 5-12 years were included in the study. Fifty patients (48\%) were evaluated for bacterial infections and 54 (52\%) for viral infections. Patients with viral infection receiving a mixture product showed earlier improvement, compared to placebo group. CARIFS scores were significantly lower in antibiotic + mixture group on day-2 and day-4, compared to antibiotic alone group $(\mathrm{p}<0.05)$. None of the patients developed any reactions or side effects to the mixture product.

Conclusions. Bee products are effective in symptomatic treatment of upper respiratory tract infections. Bee products can be considered as a good treatment option because the available drugs already used for symptomatic treatment are not cost effective and can also have serious side effects in children.

Key words: honey, propolis, royal jelly, upper respiratory tract infection.

The most common infectious disease in children is acute upper respiratory tract infection (URTI). ${ }^{1}$ Uncomplicated infections typically cause runny nose and obstruction, fever, throat pain, and cough. ${ }^{2}$ URTIs can be of either viral or bacterial origin, whose incidence markedly increase in winter months, threatening public health., ${ }^{2,3}$ Many drugs, especially antitussive drugs, are widely used for symptomatic treatment. ${ }^{4}$ Numerous adverse effects have been reported

\Yılmaz Seçilmiş

yildosec@hotmail.com

Received 6th February 2018, revised 11th June 2018, 16th October 2018, accepted 7th May 2019. in children under 6 years using antitussive, decongestant, and antihistamine drugs. ${ }^{5-8}$

The Canadian Acute Respiratory Illness and Flu Scale (CARIFS) Score was established for the first time in a study involving 220 children in 2000, in order to evaluate the severity and duration of the infection objectively. There are 18 different questions related to URTI symptoms on the scale, as families will be classified according to its seriousness. ${ }^{9}$ Honey has antibacterial and antiviral effects and is recommended by the World Health Organization (WHO) for symptomatic treatment, primarily for cough in URTIs. ${ }^{10-12}$ Propolis and royal jelly are beederived products. As a positive effect, they reduce bacterial motility and synergistically 
increase antioxidant activity with their honey component. ${ }^{13,14}$ There are studies in children using honey or propolis together or separately for URTIs. ${ }^{15-18}$

The aim of this study was to determine the effectiveness of a mixture of bee products (honey, royal jelly, propolis), as they are inexpensive (the price of the product is about one dollar), reliable, and have been used for many years for both symptoms and infection progression in URTI-infected children. To the best of our knowledge, it is the first study in which three different products were used, so that viral and bacterial infections could be evaluated separately.

\section{Material and Methods}

The study was performed prospectively. A randomized, double-blind, placebo-controlled trial procedure was used, which included two hundred patients aged 5-12 years who were diagnosed with viral and bacterial tonsillopharyngitis at Erciyes University Mustafa Eraslan-Fevzi Mercan Children's Hospital and had complaints such as fever, sore throat and a cough, during the period from 1 October 2015 to 29 February 2016. Group selection was done completely randomly by computer assignment. The computer program randomly decided a patient's allocation into one of the groups.

Fifty patients were included in four groups of volunteers of similar age range and gender. The placebo group (control) received starch and brown sugar in equal amounts with a mixture of bee products, which was prepared by a commercial company. Ninety-six patients who did not return the case form, who did not attend the control examination, who missed the case form, who did not use appropriate treatment were excluded from the study. Of the remaining 104 patients, 59 (56.7\%) were male and $45(43.3 \%)$ were female. Eleven $(11.4 \%)$ patients were 5 years old, $8(8.3 \%)$ were 6 years old, 14 (14.6\%) were 7 years old, 16 (16.6\%) were 8 years old, 14 (14.6\%) were 10 years old, 12 $(12.5 \%)$ were 11 years old, and 11 (11.4\%) were 12 years old. The products given to all patients were numbered and given in boxes of ten. For this reason, neither the doctor nor patient knew what product was used. Informed consent was received from all families.

\section{Clinical findings and follow-up}

At the time of admission, a detailed medical and allergy history of each patient was taken; it included school absenteeism, history of allergy, the number of illnesses per year, smoking at home, parents' school degrees and ages, and the number of siblings. We recorded the physical examination findings (including height and weight) and filled out the case form.

We delineated the clinical findings of each case, with CARIFS score tables (for 10 days) given to each set of parents who were included in the study. In this scoring, parents were asked to fill out these forms by asking 18 questions that included presence of a sore throat, muscular pain, fever, cough, nasal congestion or runny nose, headache, poor appetite, insomnia, irritable, unwellness, tiredness, excessive crying, need for extra care, clingy, vomiting, uninterested, lack of willingness to sleep and unamenable to play. When the complaints began, we also asked whether the child received any medication before treatment, and if any side effects occurred during that time. The doctor was seen on the first day and the fever was recorded. The answers to each question (no problem $=0$, little problem $=1$, medium problem $=2$, and large problem=3) were considered.

\section{Laboratory tests}

Five $\mathrm{ml}$ blood samples were taken from all subjects: C-reactive protein (CRP), a complete blood count (CBC), and erythrocyte sedimentation rate (ESR) were studied. The tests were carried out at Erciyes University Mustafa Gündoğdu Central Laboratory. 


\section{Bacterial and viral analyses}

A throat culture for diagnostic purposes was taken from each patient. Sheep bloody agar was used for growing beta hemolytic streptococcus group A, as it is frequently encountered as the etiology of URTIs. Nasal swab polymerase chain reaction (PCR) and Epstein-Barr viral capsid antigen immunoglobulin $\mathrm{M}$ were used to detect a viral etiology. Patients with cryptic tonsillitis, leukocytosis, and elevated CRP (plus combining these three findings) were evaluated, as bacterial infections were included in the study. Patients without bacterial infections who had normal throat cultures were excluded from the evaluation. Patients were initially divided into two groups, i.e., those with both viral and bacterial infection.

In the group of bacterial infections, half the patients took antibiotics and the other half took antibiotics and a mixture of the products. Half of the viral infection group was given a placebo; the remaining half took a mixture product. A total of 104 patients were divided into four groups.

We used paracetamol at a dose of $10 \mathrm{mg} / \mathrm{kg}$ for 6 hours or ibuprofen at a dose of $5 \mathrm{mg} / \mathrm{kg}$ for 8 hours when the body temperature exceeded $38^{\circ} \mathrm{C}$ while medical treatment was applied in the groups.

The mixture products were consumed once a day for 10 days (20 g/day for children under $30 \mathrm{~kg}, 40 \mathrm{~g} /$ day for children over $30 \mathrm{~kg}$ ). A jar mixture product consisted of $25 \mathrm{ml}$. The mixture was $96.7 \%$ honey, $3 \%$ royal jelly, and $0.03 \%$ propolis. The maximal moisture content of the honey was determined to be $20 \%$, the maximum free acidity was $50 \mathrm{mEq} / \mathrm{kg}$, the hydroxymethyl furfural (HMF) value was a maximum of $40 \mathrm{mg} / \mathrm{kg}$, the fructose and glucose content was a minimum of $60 \%$, and the sucrose content was a maximum of $5 \%$. Antibiotics and pesticides were analyzed for honey, royal jelly, and propolis to ensure they did not exert any residual risk. The research lasted 10 days, with phone calls made to parents every three days.
The study was approved by the Ethics Committee of Erciyes University (07.03.2014, No. 2014/140).

\section{Statistical analysis}

SPSS for Windows, v. 21.0 was used for statistical analysis. Normal distribution of numerical variables was evaluated by Shapiro-Wilk normality test and Q-Q graphs. Descriptive statistics were given as number of units $(\mathrm{n})$, percentage (\%), mean \pm standard deviation, mean \pm standard error, median $\left(25^{\text {th }}\right.$ $-75^{\text {th }}$ percentiles). Comparisons between the groups were performed with one-way analysis of variance (ANOVA) analysis for the normal distribution of maternal age, fever, hemoglobin, hematocrit, and platelet count variables. If there was a difference in the ANOVA results, Tukey HSD test was used as a posthoctest. Comparisons between the groups were performed with Kruskal-Wallis analysis for the non-normal distribution of the number of siblings, school absenteeism, number of illnesses per year, body weight, laboratory parameters such as $\mathrm{CBC}, \mathrm{CRP}$, anti-streptolysin O (ASO) titer and ESR. The Dunn-Bonferroni test was used as a post hoc test in case of a difference between the Kruskal-Wallis analysis. Two-way analysis of variance was used for repeated measurements of general linear models in comparison of time scores of the groups. Bonferroni test was used as a multiple comparison test. A $\mathrm{p}$ value $<0.05$ was considered statistically significant.

\section{Results}

Two hundred patients were included in the study, but 104 score tables were evaluated (52\%), including fifty-nine males (56.73\%) and 45 females (43.27\%). Those who were admitted to Erciyes University Mustafa Eraslan-Fevzi Mercan Children's Hospital between 1 October 2015 and 29 February 2016 were included in the study. Complaints identified in patients diagnosed with URTIs were fever, cough, sore throat, vomiting and nasal obstruction. 
The analysis of the answers about the secondary factors revealed that $78.1 \%$ of patients' parents did not work and $34.7 \%$ of them were primary school graduates. In addition, $77.1 \%$ of patients were found to have no allergies, but $53.5 \%$ were around smokers at home. Patients treated with the mixture product had no reaction or side effects. CBC and CRP values were measured to give an etiological evaluation to patients in the study. Laboratory values on the first day of admission to the hospital are given in Table I. There was no statistically significant difference between the groups in terms of number of siblings, school absenteeism, number of URTIs for one-year, maternal age, fever, and body weight ( $p>0.05$; data are not shown). CRP, WBCs, and neutrophil values were found to be higher in patients with a bacterial infection ( $p$ $<0.05$ ); platelet count was significantly higher in patients with viral-based URTIs ( $p<0.05$ ).

The scoring table for 10 days is presented in Table II. Measurement results of the general linear model were statistically significant ( $p$ $<0.05$ ). Clinical severity and complaints at the beginning of treatment period were similar in all four groups $(p>0.05)$. In the following days, however, in bacterial infection groups, a statistically significant decrease was found in the clinical score on the 2nd and 4th days in the group that received the mixture product and antibiotic, compared to antibiotic alone $(\mathrm{p}<0.05)$.
In the viral infection groups, while there was no statistically significant difference in the clinical score at the beginning of the treatment, statistically significant differences were found between the patients who received placebo and mixture products from the 4 th day until the last day of the treatment $(\mathrm{p}<0.05)$.

\section{Discussion}

In this study, we included children between the ages of 5 to 12 years, who presented to our pediatric emergency department with URTIs. The mixture product group showed rapid improvement in their symptoms according to CARIFS scoring, compared to the groups not using it. This finding was found to be more significant with mixture-product users with viral infections.

In the viral infection group, the symptoms of the URTI continued at a similar level for 10 days while using the placebo product, as there was no satisfactory improvement for parents and patients. There are studies reporting the effectiveness of honey, royal jelly, and propolis individually for symptomatic improvement of URTIs. ${ }^{18,27,28}$

In 2007, Paul et al. ${ }^{15}$ reported a significantly better treatment with honey versus treatment with placebo among 100 children with

Table I. Laboratory parameters according to infection and treatment groups.

\begin{tabular}{|c|c|c|c|c|c|c|}
\hline \multirow{3}{*}{ Laboratory parameters } & \multicolumn{6}{|c|}{ Infection and treatment groups } \\
\hline & \multicolumn{2}{|c|}{ Bacterial infection } & \multirow[b]{2}{*}{$\mathrm{p}-1$} & \multicolumn{2}{|c|}{ Viral infection } & \multirow[b]{2}{*}{$\mathrm{p}-2$} \\
\hline & $\begin{array}{c}\text { Antibiotic }+ \\
\text { Mixture }(n=23)\end{array}$ & $\begin{array}{l}\text { Antibiotic } \\
(\mathrm{n}=27)\end{array}$ & & Mixture $(n=25)$ & Placebo $(n=29)$ & \\
\hline Hemoglobin, g/dl & $12.94 \pm 1.3$ & $12.91 \pm 0.9$ & 0.954 & $13.04 \pm 0.8$ & $12.81 \pm 0.7$ & 0.401 \\
\hline White blood cell, $x 10^{3} / \mathrm{mm}^{3}$ & $11.4(5.9-16)$ & $14.4(6.9-16.2)$ & 0.809 & $7.2(4.6-10)$ & $8.01(5.1-11.6)$ & 0.961 \\
\hline Neutrophil, $x 10^{3} / \mathrm{mm}^{3}$ & $7.3(4.5-12.4)$ & $10.8(4.1-12.6)$ & 0.919 & $4.1(2.1-6.7)$ & $4.32(3.08-7.53)$ & 0.889 \\
\hline Lymphocyte, $x 10^{3} / \mathrm{mm}^{3}$ & $1.79(1.42-2.41)$ & $2.04(1.34-2.99)$ & 0.227 & $2.0(1.38-2.74)$ & $1.94(1.42-3.09)$ & 0.842 \\
\hline Platelet, $\times 10^{3} / \mathrm{mm}^{3}$ & $300 \pm 105$ & $299 \pm 66$ & 0.973 & $250 \pm 93$ & $231 \pm 54$ & 0.490 \\
\hline C-reactive protein, $\mathrm{g} / \mathrm{L}$ & $25.8(11.1-56.2)$ & $20.9(11-56.8)$ & 0.995 & $3.2(3.27-12.6)$ & $10.7(3.48-37.7)$ & 0.232 \\
\hline Sedimentation, mm/hour & $12(7-20.7)$ & $14.5(5.2-34.2)$ & 0.511 & $15(8-28.5)$ & $14.5(8-22.5)$ & 0.576 \\
\hline
\end{tabular}

Data are presented as mean \pm standard deviation or median $\left(25^{\text {th }}\right.$ percentile $-75^{\text {th }}$ percentile $)$ as appropriate.

p-1: comparison between "antibiotic + mixture" and "antibiotic" groups in bacterial infection

p-2: comparison between "mixture" and "placebo" groups in viral infection 
Table II. The Canadian Acute Respiratory Illness and Flu Scale (CARIFS) Score according to infection and treatment groups for 10 days.

\begin{tabular}{|c|c|c|c|c|c|c|}
\hline \multirow{3}{*}{ Days } & \multicolumn{6}{|c|}{ Infection and treatment groups } \\
\hline & \multicolumn{2}{|c|}{ Bacterial infection } & \multirow[b]{2}{*}{$\mathrm{p}-1$} & \multicolumn{2}{|c|}{ Viral infection } & \multirow[b]{2}{*}{$\mathrm{p}-2$} \\
\hline & Antibiotic + Mixture $(n=23)$ & Antibiotic $(n=27)$ & & Mixture $(n=25)$ & Placebo $(n=29)$ & \\
\hline 1 & $21.2 \pm 2.8$ & $26.5 \pm 2.62$ & 0.176 & $17.3 \pm 2.0$ & $22.4 \pm 2.9$ & 0.152 \\
\hline 2 & $16.0 \pm 2.5$ & $25.9 \pm 3.2$ & $0.017^{*}$ & $13.2 \pm 2.1$ & $19.7 \pm 2.9$ & 0.071 \\
\hline 3 & $11.9 \pm 2.2$ & $18.8 \pm 2.7$ & 0.053 & $10.1 \pm 2.1$ & $16.1 \pm 2.9$ & 0.093 \\
\hline 4 & $10.1 \pm 2.1$ & $17.0 \pm 2.4$ & $0.035^{*}$ & $6.2 \pm 1.2$ & $15.9 \pm 2.6$ & $0.001^{*}$ \\
\hline 5 & $10.7 \pm 2.7$ & $13.8 \pm 2.3$ & 0.399 & $5.0 \pm 1.2$ & $12.4 \pm 2.3$ & $0.005^{*}$ \\
\hline 6 & $7.3 \pm 2.1$ & $12.5 \pm 2.3$ & 0.088 & $5.2 \pm 1.3$ & $11.5 \pm 2.4$ & $0.020^{*}$ \\
\hline 7 & $6.2 \pm 1.7$ & $9.5 \pm 1.8$ & 0.195 & $4.5 \pm 1.1$ & $12.4 \pm 2.6$ & $0.005^{*}$ \\
\hline 8 & $6.6 \pm 1.8$ & $7.6 \pm 1.7$ & 0.678 & $4.1 \pm 1.0$ & $12.3 \pm 2.7$ & $0.004^{*}$ \\
\hline 9 & $5.1 \pm 1.7$ & $5.7 \pm 1.3$ & 0.791 & $2.8 \pm 0.9$ & $10.5 \pm 2.7$ & $0.005^{*}$ \\
\hline 10 & $3.5 \pm 1.0$ & $4.3 \pm 1.2$ & 0.600 & $1.8 \pm 0.7^{\mathrm{a}}$ & $9.9 \pm 2.6$ & $0.003^{*}$ \\
\hline
\end{tabular}

Data are presented as mean \pm standard error.

p-1: comparison between "antibiotic + mixture" and "antibiotic" groups in bacterial infection

p-2: comparison between "mixture" and "placebo" groups in viral infection

*: $\mathrm{p}<0.05$

URTIs, showing significant improvement of the symptoms of cough, although similar results were attained as compared with dextromethorphan.

Oduwole et al. ${ }^{19}$, in a study on honey use in URTIs dated 2014, reported that honey was effective against cough compared to placebo treatment and had a similar efficacy with dextromethorphan. In our study, three bee products were used as a mixture: when compared with the placebo and bacterial groups using antibiotics, not only the cough, but all symptoms (i.e. CARIFS score) improved in a significantly shorter period. Yuksel et al. ${ }^{20}$, as indicated by a review published in 2016, found that a protective mixture of royal jelly, honey, and propolis in specific ratios was also very effective, with antibacterial, antiviral, and anti-inflammatory properties. ${ }^{15}$ In 2004, Cohen et al. ${ }^{21}$ conducted a placebo-controlled double-blind study with 430 patients aged 1-5 years. They used propolis and echinacea as a mixture product and at a 12-week follow-up, a significant decrease in URTI frequency and symptoms was found. In the same study, it was reported that the duration of fever, the use of antibiotics and antipyretics, and recurrent visits to the doctor were significantly reduced. ${ }^{21}$ In our study, using all three bee products, we obtained effective results in the bacterial infection and viral infection groups. These findings support the study of Cohen et al. ${ }^{21}$ in terms of antibacterial and antiviral activity.

El-Shouny et al. ${ }^{22}$ found accelerated healing in 2-5 days in forty-one pediatric patients with URTIs, due to beta-hemolytic streptococcus throat infections when using propolis. Krol et al. ${ }^{23}$ and Speciale et al..$^{24}$ found that in vitro studies showed synergistic effects between propolis and antibiotics.

Mirzoeva et al. ${ }^{25}$ in a similar study in 1997, found combined effects with propolis and antibiotics, especially penicillin $G$ and ampicillin for URTIs. At the same time, they also found that synergistic efficacy increased with increasing doses of propolis.

In our study, the rate of infection recovery was found to be higher in the group receiving antibiotics and mixture products than those that received antibiotics alone. The results found in our study were similar to the studies of $\mathrm{Krol}^{23}$ and Mirzoeva. ${ }^{25}$ 
This study has some limitations. Initially, 200 patients were planned to be included in the study, but it was completed with 104 patients due to patient-related and technical issues.

Despite known adverse effects of antibiotics for treatment of URTIs, there are drugs like dextromethorphan, which are used to treat coughs (although they are no more effective than placebo); they are known to have numerous side effects and a higher cost, so patients decide to use herbal and bee products. ${ }^{26}$

This study has shown the effectiveness of a mixture of bee products for URTIs in children. These functional products are not only inexpensive, but have a number of beneficial effects. For this reason, we think that a mixture of bee products will be useful as an alternative treatment in URTI treatment in children.

\section{REFERENCES}

1. Green RJ. Symptomatic treatment of upper respiratory tract symptoms in children. S Afr Fam Pract 2006; 48: 38-42.

2. Pappas DE, Hendley JO, Hayden FG, Winther B. Symptom profile of common colds in school-aged children. Pediatr Infect Dis J 2008; 27: 8-11.

3. Wessels MR. Streptococcal pharyngitis. N Engl J Med 2011; 364: 648-655.

4. Centers for Disease Control and Prevention (CDC). Infant deaths associated with cough and cold medications-- two states, 2005. MMWR Morb Mortal Wkly Rep 2007; 56: 1-4.

5. Sharfstein JM, North M, Serwint JR. Over the counter but no longer under the radar- pediatric cough and cold medications. N Engl J Med 2007; 357: 2321-2324.

6. Steinman MA, Landefeld CS, Gonzales R. Predictors of broad-spectrum antibiotic prescribing for acute respiratory tract infections in adult primary care. JAMA 2003; 289: 719-725.

7. Chlabicz S, Małgorzata-Ołtarzewska A, PytelKrolczuk B. Respiratory tract infections: Diagnosis and use of antibiotics by family physicians in northeastern Poland. Int J Antimicrob Agents 2004; 23: 446-450.

8. Pichichero ME. Understanding antibiotic overuse for respiratory tract infections in children. Pediatrics 1999; 104: 1384-1388.
9. Jacobs B, Young NL, Dick PT, et al. Canadian acute respiratory illness and flu scale (CARIFS): development of a valid measure for childhood respiratory infections. J Clin Epidemiol 2000; 53: 793-799.

10. World Health Organization (WHO). Cough and cold remedies for the treatment of acute respiratory infections in young children. 2001: 1-39. WHO reference number: $\mathrm{WHO} / \mathrm{FCH} / \mathrm{CAH} / 01.02$

11. Wahdan H. Causes of the antimicrobial activity of honey. Infection 1998; 26: 26-31.

12. Lusby PE, Coombes AL, Wilkinson JM. Bactericidal activity of different honeys against pathogenic bacteria. Arch Med Res 2005; 36: 464-467.

13. Finlay BB, Falkow S. Common themes in microbial pathogenicity. Microbiol Rev 1989; 53: 210-230.

14. Tamura Y, Kijima-Tanaka M, Aoki A, Ogikubo Y, Takahashi T. Reversible expression of motility and flagella in Clostridium chauvoei and their relationship to virulence. Microbiology 1995; 141(Pt 3): 605-610.

15. Paul IM, Beiler J, McMonagle A, Shaffer ML, Duda L, Berlin CM Jr. Effect of honey, dextromethorphan, and no treatment on nocturnal cough and sleep quality for coughing children and their parents. Arch Pediatr Adolesc Med 2007; 161: 1140-1146.

16. Focht J, Hansen S, Nielsen JV, Van den Berg-Segers A, Riezler R. Bactericidal effect of propolis in vitro against agents causing upper respiratory tract infections. Arzneimittelforschung 1993; 43: 921-923.

17. Park YK, Koo MH, Abreu JA, Ikegaki M, Cury JA, Rosalen PL. Antimicrobial activity of propolis on oral microorganisms. Curr Microbiol 1998; 36: 24-28.

18. Shadkam MN, Mozaffari-Khosravi H, Mozayan MR. A comparison of the effect of honey, dextromethorphan, and diphenhydramine on nightly cough and sleep quality in children and their parents. J Altern Complement Med 2010; 16: 787-793.

19. Oduwole O, Meremikwu MM, Oyo-Ita A, Udoh EE. Honey for acute cough in children. Cochrane Database Syst Rev 2014; 9: 401-444.

20. Yuksel S, Akyol S. The consumption of propolis and royal jelly in preventing upper respiratory tract infections and as dietary supplementation in children. J Intercult Ethnopharmacol 2016; 5: 308311.

21. Cohen HA, Varsano I, Kahan E, Sarrell EM, Uziel Y. Effectiveness of an herbal preparation containing echinacea, propolis, and vitamin $\mathrm{C}$ in preventing respiratory tract infections in children: a randomized, double-blind, placebo-controlled, multicenter study. Arch Pediatr Adolesc Med 2004; 158: 217-221. 
22. El-Shouny W, Muagam F, Sadik Z, Walaa H. Antimicrobial activity of propolis extract on URT infections in pediatric patients admitted to AlThowrah Hospital, Hodeidah City, Yemen. World J Med Sci 2012; 7: 172-177.

23. Krol W, Scheller S, Shani J, Pietsz G, Czuba Z. Synergistic effect of ethanolic extract of propolis and antibiotics on the growth of staphylococcus aureus. Arzneimittelforschung 1993; 43: 607-609.

24. Speciale A, Costanzo R, Puglisi S, et al. Antibacterial activity of propolis and its active principles alone and in combination with macrolides, beta-lactams and fluoroquinolones against microorganisms responsible for respiratory infections. J Chemother 2006; 18: 164-171.
25. Mirzoeva OK, Grishanin RN, Calder PC. Antimicrobial action of propolis and some of its components: the effects on growth, membrane potential and motility of bacteria. Microbiol Res 1997; 152: 239-246.

26. Mangione-Smith R, Stivers T, Elliott M, McDonald L, Heritage J. Online commentary during the physical examination: A communication tool for avoiding inappropriate antibiotic prescribing? Soc Sci Med 2003; 56: 313-320.

27. Carr RR, Nahata M. Complementary and alternative medicine for upper-respiratory tract infection in children. Am J Health Syst Pharm 2006; 63: 33-39.

28. Goldman RD. Honey for treatment of cough in children. Can Fam Physician 2014; 60: 1107-1110. 